\title{
Global Properties of Evolutional Lotka-Volterra System
}

\author{
Masafumi Yoshino ${ }^{1}$, Yoshinari Tanaka ${ }^{2}$ \\ ${ }^{1}$ Department of Mathematics, Graduate School of Science, Hiroshima University, Higashi-Hiroshima, Japan \\ ${ }^{2}$ Research Center for Environmental Risk, National Institute for Environmental Studies, Tsukuba, Japan \\ Email: yoshinom@hiroshima-u.ac.jp,ytanaka@nies.go.jp
}

Received November 9, 2013; revised December 9, 2013; accepted December 15, 2013

Copyright (C) 2013 Masafumi Yoshino, Yoshinari Tanaka. This is an open access article distributed under the Creative Commons Attribution License, which permits unrestricted use, distribution, and reproduction in any medium, provided the original work is properly cited. In accordance of the Creative Commons Attribution License all Copyrights (C) 2013 are reserved for SCIRP and the owner of the intellectual property Masafumi Yoshino, Yoshinari Tanaka. All Copyright (C) 2013 are guarded by law and by SCIRP as a guardian.

\section{ABSTRACT}

We will study global properties of evolutional Lotka-Volterra system. We assume that the predatory efficiency is a function of a character of species whose evolution obeys a quantitative genetic model. We will show that the structure of a solution is rather different from that of a non-evolutional system. We will analytically show new ecological features of the dynamics.

Keywords: Lotka-Volterra System; Global Dynamics; Evolution

\section{Introduction}

In this paper, we study global behavior of an evolutional Lotka-Volterra system for three species

$$
\begin{gathered}
\frac{\mathrm{d} N_{1}}{\mathrm{~d} t}=r N_{1}\left(1-N_{1} / K\right)-\frac{a_{2} N_{1} N_{2}}{1+a_{2} h_{2} N_{1}}, \\
\frac{\mathrm{d} N_{2}}{\mathrm{~d} t}=\frac{c_{2} a_{2} N_{1} N_{2}}{1+a_{2} h_{2} N_{1}}-d_{2} N_{2}-\frac{a_{3} N_{2} N_{3}}{1+a_{3} h_{3} N_{2}}, \\
\frac{\mathrm{d} N_{3}}{\mathrm{~d} t}=\frac{c_{3} a_{3} N_{2} N_{3}}{1+a_{3} h_{3} N_{2}}-d_{3} N_{3}, \\
\frac{\mathrm{d} z_{2}}{\mathrm{~d} t}=-g \delta^{-1}\left(z_{2}-\hat{z}_{2}\right)-\frac{g N_{3} a_{3}^{\prime}}{\left(1+a_{3} h_{3} N_{2}\right)^{2}},
\end{gathered}
$$

for the unknown quantities $N_{j}(t)(j=1,2,3)$ and $z_{2}$ which are the population of $j$ th species and the mean character value of the second species, respectively. Here $r>0, K>0, h_{2}>0, h_{3}>0, c_{2}>0, c_{3}>0$ are certain constants, and $d_{2}>0$ and $d_{3}>0$ are death rate of the second and the third species, respectively. The quantities $a_{2}>0$ and $a_{3}>0$ are the predatory efficiency of the second and the third species, respectively. The number $\hat{z}_{2}$ is the mean character value of the second species with minimal cost. The quantity $g>0$ is the additive genetic variance and $\delta>0$ is the cost of evolution, namely, if $\delta$ decreases, then the cost increases.
The effect of evolution is expressed in terms of (1.4) and the condition that the predatory efficiency $a_{3} \equiv a_{3}\left(z_{2}-\hat{z}_{2}\right)$ is given by

$$
a_{3} \equiv a_{3}\left(z_{2}-\hat{z}_{2}\right)=a_{3}^{0}-a\left(z_{2}-\hat{z}_{2}\right),
$$

where $a_{3}^{0}>0$ is a given constant and $0 \leq a(z) \leq a_{3}^{0}$ is a function of $z$. An example of $a_{3}$ is given by (C.1) in Section 3. Equation (1.4) follows the quantitative genetical model (cf. [1-5]. See also Section 7). The evolutional Lotka-Volterra system for two species was studied in [3], where rather detailed numerical analysis was made. As for the system for three species, very little is known as to global behavior of solutions even from a numerical point of view. In this paper, we shall make the analytical study of evolutional Lotka-Volterra model for three species and show several new phenomena caused by evolution. We also refer [6] as to non-evolutional case.

Let $\mathbb{R}_{+}:=\{t \in \mathbb{R} ; t \geq 0\}$. Let $t_{0}$ and $N_{i} \in \mathbb{R}_{+}(i=1,2,3)$ and $z_{2}^{0}$ be given. We first prove that (1.1)-(1.4) with the initial condition

$$
\begin{aligned}
& \left.\left(N_{1}, N_{2}, N_{3}\right)\right|_{t=t_{0}}=\left(N_{1}^{0}, N_{2}^{0}, N_{3}^{0}\right) \in \mathbb{R}_{+}^{3}, \\
& \left.z_{2}\right|_{t=t_{0}}=z_{2}^{0},
\end{aligned}
$$

have unique smooth time global solution. (cf. Theorem 2 ). Then, in terms of estimate of a solution obtained in the proof of Theorem 2, we study behaviors of a solution 
related to evolution. Indeed, we will show that the behavior of a solution near the equilibrium point is different from those in the case of tea-cup attractors for a nonevolutional system. Namely, the decay of the predator $N_{3}$ starts before the quantity $N_{2}$ becomes small because the predatory efficiency $a_{3}$ tends to zero, by evolution. We remark that although $N_{3} / N_{2}$ plays an important role in the non-evolutional system near equilibrium point, the quantity $\sqrt{N_{3}} / N_{2}$ is crucial in the evolutional one. This is because the quantity $\sqrt{N_{3}} / N_{2}$ is related with the dynamics of evolution. We remark that the effect of evolution in our system is intermittent in the sense that in some subdomain of the phase space flactuations of pray $N_{1}, N_{2}$ occur as in the case of non-evolutional model, while in other subdomain, evolution stabilizes large fluctuations of $N_{2}$ and $N_{3}$. We also discuss the role of $\gamma$ in (C.1), which is related with the sensitivity of evolution to the character bias $z_{2}-\hat{z}_{2}$. (cf. Lemma 3 and Section 4 for the case of a linear efficiency). In Section 5, we study the uniform convergence of solutions of an evolutional system as the cost of evolution tends to infinity, i.e., $\delta$ decreases to zero.

\section{Time Global Solution}

We shall study the global existence and uniqueness of a solution of the initial value problem. We assume that $0 \leq a(z) \leq a_{3}^{0}$ is the twice continuously differentiable function which satisfies

$$
a(0)=0, a^{\prime}(0)=0,
$$

for some $a_{3}^{0}>0$. Moreover we suppose that there exist $\ell_{0}>0$ and $\ell_{1}>0$ such that

$$
a(z)=a_{3}^{0}, \forall z \geq \ell_{0}, z \leq-\ell_{1} .
$$

The following local existence and uniqueness theorem is well known.

THEOREM 2.1. Assume (2.1) and (2.2). Then there exists $a \quad \delta>0$ such that the system of Equations (1.1)(1.4) with the initial conditions (1.6) has a unique continuously differentiable solution $\left(N_{1}, N_{2}, N_{3}\right), z_{2}$ in $\left|t-t_{0}\right|<\delta$.

In the following we study the existence of a global solution. We require the condition

$$
0<N_{1}^{0}<K, N_{2}^{0}>0, N_{3}^{0}>0,-\ell_{1}<z_{2}^{0}-\hat{z}_{2}<\ell_{0} .
$$

Remark. If $N_{i}^{0}=0$ for some $j$, then, by the uniqueness, any solution of (1.1)-(1.4) satisfies $N_{i} \equiv 0$. Hence it reduces to a system with less unknown quantities. Note that we avoid this case in (2.3).

We have

THEOREM 2.2. Suppose that (2.3) is satisfied. Then the system of Equations (1.1)-(1.4) with the initial condition (1.6) has a unique global solution in $t_{0} \leq t<\infty$.

Proof. First we will show the apriori estimate $N_{1}(t)<K$ for all $t$. Suppose that this is not true. Then, by the continuity of $N_{1}(t)$ and $N_{1}\left(t_{0}\right)=N_{1}^{0}<K$ in (2.3) we can take the smallest time $t_{1}>t_{0}$ such that $N_{1}\left(t_{1}\right)=K$. Assume that $N_{2}\left(t_{1}\right)=0$. If we set $N_{2}=0$ in (1.1)-(1.4), then we have

$$
\begin{aligned}
\frac{\mathrm{d} N_{1}}{\mathrm{~d} t} & =r N_{1}\left(1-\frac{N_{1}}{K}\right), \frac{\mathrm{d} N_{3}}{\mathrm{~d} t}=-d_{3} N_{3}, \\
\frac{\mathrm{d} z_{2}}{\mathrm{~d} t} & =-\delta^{-1} g\left(z_{2}-\hat{z}_{2}\right)-g N_{3} a_{3}^{\prime} .
\end{aligned}
$$

By the local existence and uniqueness theorem, Equations (2.4) with the initial condition $\left.\left(N_{1}, N_{3}, z_{2}\right)\right|_{t=t_{1}}=\left(K, N_{3}\left(t_{1}\right), z_{2}^{0}\right)$ has a unique solution. We denote the solution by $\left(\tilde{N}_{1}, \tilde{N}_{3}, \tilde{z}_{2}\right)$. Then (1.1)-(1.4) with the initial value $\left(K, 0, N_{3}\left(t_{1}\right), z_{2}^{0}\right)$ at $t=t_{1}$ has a solution $\left(\tilde{N}_{1}, \tilde{N}_{3}, \tilde{z}_{2}\right)$ By the uniqueness of the solution we obtain $\left(N_{1}, N_{2}, N_{3}, z_{2}\right)=\left(\tilde{N}_{1}, 0, \tilde{N}_{3}, \tilde{z}_{2}\right)$. It follows that $N_{2}(t) \equiv 0$. Because $N_{2}\left(t_{0}\right) \neq 0$ by (2.3), we have a contradiction. Hence we have $N_{2}\left(t_{1}\right)>0$. By the continuity of $N_{2}(t)$ one may assume that $N_{2}(t)>0$ in a sufficiently small neighborhood of $t_{1}$. Then, the second term in the right hand side of (1.1) satisfies $a_{2} N_{1} N_{2} /\left(1+a_{2} h_{2} N_{1}\right)>0$ in a sufficiently small neighborhood of $t_{1}$. On the other hand, since $r N_{1}\left(1-N_{1} / K_{1}\right)$ can be made arbitrarily small by taking a neighborhood of small, it follows that $\left(\mathrm{d} N_{1} / \mathrm{d} t\right)<0$ there. Hence $N_{1}$ is a decreasing function. This contradicts to $N_{1}\left(t_{1}\right)=K$. Therefore, there is not $t_{1}$ such that $N_{1}(t)=K$, which shows the desired estimate.

Next we will estimate $N_{2}$ from the above. Take $\varepsilon>0$ that $\varepsilon c_{2} \leq 1$, and add $\varepsilon$ times (1.2) to (1.1). Then we have

$$
\begin{aligned}
& \frac{\mathrm{d} N_{1}}{\mathrm{~d} t}+\varepsilon \frac{\mathrm{d} N_{2}}{\mathrm{~d} t}=r N_{1}\left(1-N_{1} K^{-1}\right)-\frac{a_{2} N_{1} N_{2}}{1+a_{2} h_{2} N_{1}} \\
& +\varepsilon N_{2}\left(\frac{c_{2} a_{2} N_{1}}{1+a_{2} h_{2} N_{1}}-d_{2}\right)-\varepsilon \frac{a_{3} N_{3} N_{2}}{1+a_{3} h_{3} N_{2}} \\
& \leq r N_{1}\left(1-N_{1} K^{-1}\right)-d_{2} N_{2} \varepsilon=-d_{2}\left(N_{1}+\varepsilon N_{2}\right)+f(t),
\end{aligned}
$$

where $f(t)=r N_{1}\left(1-N_{1} K^{-1}\right)+d_{2} N_{1} \geq 0$.

Hence, by setting $v(t)=N_{1}+N_{2}$ we obtain

$$
\frac{\mathrm{d} v}{\mathrm{~d} t}+d_{2} \leq f(t)
$$

Multiplying $\mathrm{e}^{d_{2} t}$ to both sides, and integrating from $t_{0}$ to $t, t>t_{0}$, we obtain

$$
\begin{aligned}
\int_{t_{0}}^{t} \frac{\mathrm{d}}{\mathrm{d} s}\left(\mathrm{e}^{d_{2} s}\right) v \mathrm{~d} s & =\mathrm{e}^{d_{2} t} v(t)-\mathrm{e}^{d_{2} t_{0}} v\left(t_{0}\right) \\
& \leq\left|\int_{t_{0}}^{t} f(s) \mathrm{e}^{d_{2} s} \mathrm{~d} s\right| .
\end{aligned}
$$


By the apriori estimate there exists $M>0$ depending only on $r, K$ and $d_{2}$ such that $f(s) \leq M$. Hence we have

$$
\begin{aligned}
v(t) & \leq \mathrm{e}^{d_{2}\left(t_{0}-t\right)} v\left(t_{0}\right)+\left|\int_{t_{0}}^{t} f(s) \mathrm{e}^{d_{2}(s-t)} \mathrm{d} s\right| \\
& \leq \mathrm{e}^{d_{2}\left(t_{0}-t\right)} v\left(t_{0}\right)+M\left|\int_{t_{0}}^{t} \mathrm{e}^{d_{2}(s-t)} \mathrm{d} s\right| .
\end{aligned}
$$

Because $\left|\int_{t_{0}}^{t} \mathrm{e}^{d_{2}(s-t)} \mathrm{d} s\right| \leq d_{2}^{-1}$, we obtain

$$
v(t) \leq \mathrm{e}^{d_{2}\left(t_{0}-t\right)} v\left(t_{0}\right)+M d_{2}^{-1} \leq v\left(t_{0}\right)+M d_{2}^{-1} .
$$

It follows that, for $t>t_{0}$

$$
N_{2}(t) \leq \varepsilon^{-1}\left(M d_{2}^{-1}+N_{1}\left(t_{0}\right)+\varepsilon N_{2}\left(t_{0}\right)\right) .
$$

Note that the right hand side quantity depends on the initial value and the equation and depends neither on $\delta>$ 0 nor on $g>0$.

We make the same argument for $N_{3}(t)$. Take $\varepsilon$ so that $\varepsilon c_{3} \leq 1$, and add $\varepsilon$ times (1.3) to (1.2). Then we have

$$
\begin{aligned}
\frac{\mathrm{d} N_{2}}{\mathrm{~d} t}+\varepsilon \frac{\mathrm{d} N_{3}}{\mathrm{~d} t} & \leq N_{2}\left(\frac{c_{2} a_{2} N_{1}}{1+a_{2} h_{2} N_{1}}-d_{2}\right)-d_{3} \varepsilon N_{3} \\
& =-d_{3}\left(N_{2}+\varepsilon N_{3}\right)+f(t),
\end{aligned}
$$

where

$$
f(t)=N_{2}\left(\frac{c_{2} a_{2} N_{1}}{1+a_{2} h_{2} N_{1}}-d_{2}+d_{3}\right) .
$$

By setting $v(t):=N_{2}+\varepsilon N_{3}$ we obtain the equation $\mathrm{d} v / \mathrm{d} t \leq-d_{3} v+f$. Because this equation has a similar form as in the case $N_{2}(t)$, we can choose a constant $M_{1}>0$ depending only on $r, K, d_{2}, d_{3}$ and the initial values so that $f(t) \leq M_{1}$. Then we argue in the same way and we obtain

$$
v(t) \leq M_{1} d_{3}^{-1}+N_{2}\left(t_{0}\right)+\varepsilon N_{3}\left(t_{0}\right)
$$

In view of the definition of $v$ we have

$$
N_{3}(t) \leq \varepsilon^{-1}\left(M_{1} d_{3}^{-1}+N_{2}\left(t_{0}\right)+\varepsilon N_{3}\left(t_{0}\right)\right) .
$$

Next we will estimate $N_{1}(t)$ from the below. By the estimates of $N_{1}$ and $N_{2}$ from the above there exists $L_{2}$ such that $N_{1}(t) \leq K, N_{2} \leq L_{2}$. It follows that

$$
\begin{aligned}
\frac{\mathrm{d} N_{1}}{\mathrm{~d} t} & =r N_{1}\left(1-N_{1} K^{-1}\right)-\frac{N_{2} a_{2} N_{1}}{1+a_{2} h_{2} N_{2}} \\
& >-\frac{N_{2} a_{2} N_{1}}{1+a_{2} h_{2} N_{2}}>-a_{2} N_{1} N_{2} \geq-a_{2} N_{1} L_{2} .
\end{aligned}
$$

By integrating from $t_{0}$ to $t$ we obtain

$$
N_{1}(t) \geq N_{1}\left(t_{0}\right) \exp \left(-a_{2} L_{2}\left|t-t_{0}\right|\right) .
$$

We will estimate $N_{2}$ from the below. There exist con- stants $A_{3}>0, L_{3}>0$ depending on the equation and the initial values such that, $\left|a_{3}\right| \leq A_{3}, N_{3} \leq L_{3}$. Hence we have

$$
\begin{aligned}
\frac{\mathrm{d} N_{2}}{\mathrm{~d} t} & =\frac{c_{2} N_{2} a_{2} N_{1}}{1+a_{2} h_{2} N_{1}}-d_{2} N_{2}-\frac{N_{2} a_{3} N_{3}}{1+a_{3} h_{3} N_{2}} \\
& >-d_{2} N_{2}-N_{2} a_{3} N_{3} \geq-d_{2} N_{2}-N_{2} A_{3} L_{3} .
\end{aligned}
$$

By integrating the inequality from $t_{0}$ to $t$ we obtain

$$
N_{2}(t) \geq N_{2}\left(t_{0}\right) \exp \left(-\left(d_{2}+A_{3} L_{3}\right)\left|t-t_{0}\right|\right) \text {. }
$$

The estimate of $N_{3}$ from the below can be shown by simple computations.

$$
N_{3}(t) \geq N_{3}\left(t_{0}\right) \exp \left(-d_{3}\left|t-t_{0}\right|\right) .
$$

Next we will prove

$$
-\ell_{1}<z_{2}(t)-\hat{z}_{2}<\ell_{0} .
$$

Indeed, we have (2.16) for $t=t_{0}$ by the initial condition. It follows that if $t-t_{0}$ is sufficiently small, then (2.16) holds true.

In order to prove (2.16) we assume that there exists $t_{1}$ such that either $z_{2}\left(t_{1}\right)-\hat{z}_{2}=\ell_{0}$ or $z_{2}\left(t_{1}\right)-\hat{z}_{2}=\ell_{1}$ holds and we show the contradiction. For the sake of simplicity let us assume the former case holds. The latter case can be treated in the same way.By the estimate of $N_{3}(t)$ from the above we have, for any $\varepsilon, 0<\varepsilon<g \ell_{0} /(2 \delta)$ there exists a neighborhood $V$ of $\ell_{0}$ such that if $z_{2}-\hat{z}_{2} \in V$, then $z_{2}(t)-\hat{z}_{2}>\ell_{0} / 2$ and $g\left|N_{3} a_{3}^{\prime}\left(z_{2}-\hat{z}_{2}\right)\right|<\varepsilon$ hold. Hence we have

$$
\begin{aligned}
\frac{\mathrm{d} z_{2}}{\mathrm{~d} t} & =-\frac{g}{\delta}\left(z_{2}-\hat{z}_{2}\right)-g \frac{N_{3} a_{3}^{\prime}\left(z_{2}-\hat{z}_{2}\right)}{\left(1+a_{3} h_{3} N_{2}\right)^{2}} \\
& <-\frac{g}{\delta}\left(z_{2}-\hat{z}_{2}\right)+\varepsilon .
\end{aligned}
$$

If $z_{2}-\hat{z}_{2} \in V$, then the right hand side of (2.17) is negative. Therefore $z_{2}(t)-\hat{z}_{2}$ is decreasing near $t=t_{1}$. This implies that $z_{2}(t)-\hat{z}_{2}<\ell_{0}$ does not tend to $\ell_{0}$ when $t \rightarrow t_{1}$. Because $z_{2}(t)$ is continuous, we have $z_{2}\left(t_{1}\right)-\hat{z}_{2} \neq \ell_{0}$. This is a contradiction. Hence we have the desired estimate.

We shall prove the existence of a global solution. Set $N(t)=\left(N_{1}, N_{2}, N_{3}\right)$ and let $t_{0} \leq t<\alpha$ be the maximal interval for which $N(t)$ and $z_{2}(t)$ are defined. If $\alpha=\infty$, then we are done. Assume that $\alpha<\infty$. We will show that the limits $\lambda_{j}:=\lim _{t \rightarrow \alpha} N_{j}(t)$ and $\beta:=\lim _{t \rightarrow \alpha} z_{2}(t)$ exist. We set $F\left(N, z_{2}\right)=F=\left(F_{1}, F_{2}, F_{3}, F_{4}\right)$, where $F_{j}$ is the right hand sides of the Equations (1.1)-(1.4), respectively. We write (1.1)-(1.4) into an equivalent system of integral equations

$$
N_{j}(t)-N_{j}\left(t_{0}\right)=\int_{t_{0}}^{t} F_{j}\left(N, z_{2}\right) \mathrm{d} \sigma .
$$


By the apriori estimates from the above,

$F_{j}\left(N(t), z_{2}(t)\right)$ is bounded on $t_{0} \leq t \leq \alpha$. Hence there exists $M$ such that $N_{j}(t)-N_{j}(s) \leq M|t-s|$. It follows that the limit $\lambda_{j} \equiv \lim _{t \rightarrow \alpha} N_{j}(t)$ exists. If we define $\lambda_{j}:=N_{j}(\alpha)>0$, then $N_{j}(t)$ is continuous up to $t=\alpha$. We will show that it is $C^{1}$. For this purpose it is sufficient to show that $\lim _{t, s \rightarrow \alpha}\left(N_{j}^{\prime}(t)-N_{j}^{\prime}(s)\right)=0$. We note that $F_{j}\left(N, z_{2}\right)$ is Lipschitz continuous in each variable because we have apriori estimates of $N$ and $z_{2}$. Namely there exists $C>0$ independent of $N$ and $z_{2}$ such that

$$
\left|F_{j}\left(N, z_{2}\right)-F_{j}\left(\tilde{N}, \tilde{z}_{2}\right)\right| \leq C|N-\tilde{N}|+C\left|z_{2}-\tilde{z}_{2}\right| .
$$

Hence, by (1.1)-(1.4) we have

$$
\left|N_{j}^{\prime}(t)-N_{j}^{\prime}(s)\right| \leq C|N(t)-N(s)|+C\left|z_{2}(t)-z_{2}(\mathrm{~s})\right| .
$$

This proves the assertion. We can similarly prove for $z_{2}(t)$.We can solve (1.1)-(1.4) with the initial values $\lambda_{j}$ and $z_{2}^{0}, z_{2}^{0}:=\lim _{t \rightarrow \alpha} z_{2}(t)$ at $t=\alpha$. Then by the unique existence of the solution we can extend $N(t)$ and $z_{2}(t)$ to some neighborhood of $t=\alpha$. This contradicts to the definition of $\alpha$. Hence we have $\alpha=\infty$. This ends the proof.

Remark. 1) We remark that the apriori estimate of a solution does not depend on the cost of evolution $0<\delta<\infty$ and the additive genetic variance $g>0$. This means that the evolution of a character has little effect to the bound of sum of populations of three species.

2) As a corollary to Theorem 2 we see that if there is no effect of evolution, i.e., $a_{3}\left(z_{2}-\hat{z}_{3}\right) \equiv a_{3}^{0}$, then (1.1)(1.3) with the initial condition (1.6) has a unique global solution. Indeed, (1.1)-(1.4) can be split into (1.1)-(1.4), $\mathrm{d} z_{2} / \mathrm{d} t=-g \delta^{-1}\left(z_{2}-\hat{z}_{2}\right)$. The latter equation can be integrated. In view of the uniqueness of the solution of (1.1)-(1.4) we see that (1.1)-(1.3) has a unique solution.

\section{Intermittency of Evolution Effect}

We shall study the effect of evolution to dynamics of (1.1)-(1.4).More precisely, we will study how the dynamics of (1.4) is related with that of (1.1)-(1.3).By setting $z=z_{2}-\hat{z}_{2}$ we write (1.4) in the form

$$
\frac{\mathrm{d} z}{\mathrm{~d} t}=-\frac{g z}{\delta}-\frac{g N_{3} a_{3}^{\prime}(z)}{\left(1+a_{3}(z) h_{3} N_{2}\right)^{2}} .
$$

Let $\gamma \geq 1$ be an integer and $\ell_{0}>0$ and $0<\eta \ll 1$ be constants. We assume

$$
\begin{aligned}
& a_{3}(z)=a_{3}^{0}\left(\ell_{0}^{2 \gamma}-z^{2 \gamma}\right), \text { for }|z|<\ell_{0}-\eta, \\
& a_{3}(z) \equiv 0, \text { for }|z| \geq \ell_{0} .
\end{aligned}
$$

We also assume that $a_{3}(z)$ is twice continuously differentiable and nonnegative in the closed intervals $\left[\ell_{0}-\eta, \ell_{0}\right]$ and $\left[-\ell_{0},-\ell_{0}+\eta\right]$. If we denote the right-hand side of (3.1) by $f(z)$, then, by (C.1) we have

$$
f(z)=\frac{2 \gamma g z N_{3} a_{3}^{0} z^{2 \gamma-2}}{\left(1+a_{3}^{0}\left(\ell_{0}^{2 \gamma}-z^{2 \gamma}\right) h_{3} N_{2}\right)^{2}}-\frac{g z}{\delta},
$$

on $|z| \leq \ell_{0}-\eta$. We define $\tilde{f}(z)$ on $|z| \leq \ell_{0}$ by the right-hand side of (3.2). Define, for $\gamma \geq 1$,

$$
v:=\sqrt{N_{3}} / N_{2}, K_{\gamma}:=\sqrt{2 \gamma \delta} / \sqrt{a_{3}^{0}} h_{3} \ell_{0}^{\gamma+1} .
$$

We first study the behavior of $\tilde{f}(z)$.

LEMMA 3.1. 1) Assume $\gamma \geq 2$. Suppose that $N_{3}<1 /\left(2 \gamma a_{3}^{0} \ell_{0}^{2 \gamma-2} \delta\right)$. Then $\tilde{f}(z)$ has a unique zero $z$ $=0$ in the interval $\left(-\ell_{0}, \ell_{0}\right)$ and $\tilde{f}(z) / z$ is negative on $\left(-\ell_{0}, \ell_{0}\right) \backslash\{0\}$.

Assume $N_{3} \geq 1 /\left(2 \gamma a_{3}^{0} \ell_{0}^{2 \gamma-2} \delta\right)$. Then $\tilde{f}(z)$ has simple zeros, $\pm z_{0}$ and 0 on $\left[-\ell_{0}, \ell_{0}\right]$. The function $\tilde{f}(z) / z$ is negative on the intervals $\left(-z_{0}, 0\right)$ and $\left(0, z_{0}\right)$, while it is positive on $\left(-\ell_{0},-z_{0}\right)$ and $\left(z_{0}, \ell_{0}\right)$. (cf. Figure 1).

Moreover, there exists $\epsilon_{1}>0$ such that $z_{0}$ has an asymptotic behavior

$$
z_{0} / \ell_{0}=1-K_{\gamma} v\left(2 \gamma+(\gamma-1) K_{\gamma} v\right)^{-1}+O\left(\left(K_{\gamma} v\right)^{2}, N_{2}^{-1}\right),
$$

when $K_{\gamma} v \rightarrow 0, N_{2} \geq \epsilon_{1}$. Similarly we have

$$
\begin{aligned}
\frac{z_{0}}{\ell_{0}}= & \left(1+1 / a_{3}^{0} \ell_{0}^{2 \gamma} h_{3} N_{2}\right)^{1 /(\gamma-1)} \times\left(K_{\gamma} v\right)^{-1 /(\gamma-1)} \\
& +O\left(\left(K_{\gamma} v\right)^{-(1+2 \gamma) / \gamma-1}\right),
\end{aligned}
$$

when $K_{\gamma} v \rightarrow \infty$.

2) Assume $\gamma=1$. If $1+\left(a_{3}^{0} \ell_{0}^{2} h_{3} N_{2}\right)^{-1}>K_{1} v$, then $\tilde{f}(z)$ has simple zeros, $\pm z_{0}$ and 0 on $\left(-\ell_{0}, \ell_{0}\right)$.

The function $\tilde{f}(z) / z$ is negative on the intervals $\left(-z_{0}, 0\right)$ and $\left(0, z_{0}\right)$, while it is positive on $\left(-\ell_{0},-z_{0}\right)$ and $\left(z_{0}, \ell_{0}\right)$. (cf. Figure 1).

If $1+\left(a_{3}^{0} \ell_{0}^{2} h_{3} N_{2}\right)^{-1} \leq K_{1} v$, then $\tilde{f}(z)$ has a unique zero $z=0$ in $\left(-\ell_{0}, \ell_{0}\right)$ and $\tilde{f}(z) / z$ is positive on $\left(-\ell_{0}, \ell_{0}\right) \backslash\{0\}$. (cf. Figure 2).

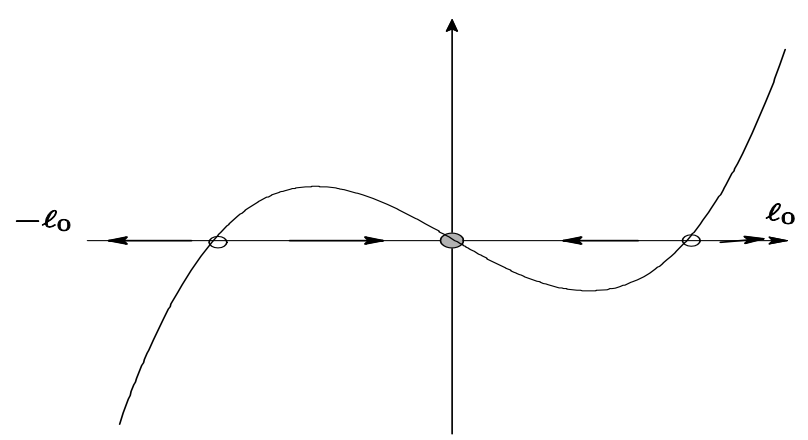

Figure 1. $\gamma \geq 2$. 
Proof. We divide the proof into 5 steps.

Step 1. By (C.1) we have

$\delta f / g z$

$$
\begin{aligned}
= & \left(1+a_{3} h_{3} N_{2}\right)^{-2}\left(2 \gamma N_{3} a_{3}^{0} \delta z^{2 \gamma-2}-\left(1+a_{3} h_{3} N_{2}\right)^{2}\right) \\
= & -\left(a_{3}^{0}\right)^{2} h_{3}^{2} N_{2}^{2}\left(1+a_{3} h_{3} N_{2}\right)^{-2} \\
& \left(K_{\gamma} \ell_{0}^{\gamma+1} v z^{\gamma-1}+\left(a_{3}^{0} h_{3} N_{2}\right)^{-1}+\ell_{0}^{2 \gamma}-z^{2 \gamma}\right) \\
& \times\left(-K_{\gamma} \ell_{0}^{\gamma+1} v z^{\gamma-1}+\left(a_{3}^{0} h_{3} N_{2}\right)^{-1}+\ell_{0}^{2 \gamma}-z^{2 \gamma}\right) .
\end{aligned}
$$

Set

$$
A^{\gamma}=1 / \ell_{0}^{2 \gamma} a_{3}^{0} h_{3} N_{2}, \zeta=z / \ell_{0},
$$

and define

$$
g_{ \pm}(\zeta)=\zeta^{2 \gamma} \pm v K_{\gamma} \zeta^{\gamma-1}-1-A_{\gamma}
$$

We consider the zeros and the sign of $g_{ \pm}(\zeta)$ in the interval $-1 \leq \zeta \leq 1$.

Step 2. First we consider the case $\gamma \geq 2$. Assume that $\gamma$ is an odd integer. Because $\gamma-1$ is an even integer, we have $g_{-}(\zeta)=g_{-}(-\zeta)$. We have

$g_{-}(1)=-v K_{\gamma}-A_{\gamma}<0$ and $g_{-}(0) \leq-1-A_{\gamma}<0$. Because we easily see that $g_{-}(\zeta)<0$ if $0 \leq \zeta \leq 1$, it follows that $g_{-}$has no zero point on $[-1,1]$.

In order to study the zero of $g_{+}$in $(0,1)$, note $g_{+}(0)<-1$. One easily see that the assumption

$N_{3} \geq 1 /\left(2 \gamma a_{3}^{0} \ell_{0}{ }^{2 \gamma-2} \delta\right)$ is equivalent to

$g_{+}(1)=v K_{\gamma}-A_{\gamma} \geq 0$. Because $g_{+}^{\prime}(\zeta)>0$ on $(0,1]$, we see that $g_{+}(\zeta)$ has only one zero point in the interval $(0,1]$ if $N_{3} \geq 1 /\left(2 \gamma a_{3}^{0} \ell_{0}^{2 \gamma-2} \delta\right)$. In view of (3.6) we conclude that $\tilde{f}$ has zero points $\pm z_{0}$ and 0 in the interval $[-1,1]$ for some $0<z_{0}<1$. It is also clear that if the opposite inequality $N_{3}<1 /\left(2 \gamma a_{3}^{0} \ell_{0}{ }^{2 \gamma-2} \delta\right)$ holds, then $\tilde{f}(z) / z<0$ on $[-1,1] \backslash\{0\}$.

Next we consider the case $\gamma$ is even. By the same way as in the odd case, we have $g_{+}(0)<-1$, $g_{+}(-1)<-v K_{\gamma}<0$ and $g_{+}(1)=v K_{\gamma}-A_{\gamma} \geq 0$. Since $g_{+}$is strictly increasing on $(0,1)$, there exists unique $\zeta_{0}$, $0<\zeta_{0} \leq 1$ such that $g_{+}\left(\zeta_{0}\right)=0$. In order to show that $g_{+}(\zeta)$ has no zero on $(-1,0)$, we note

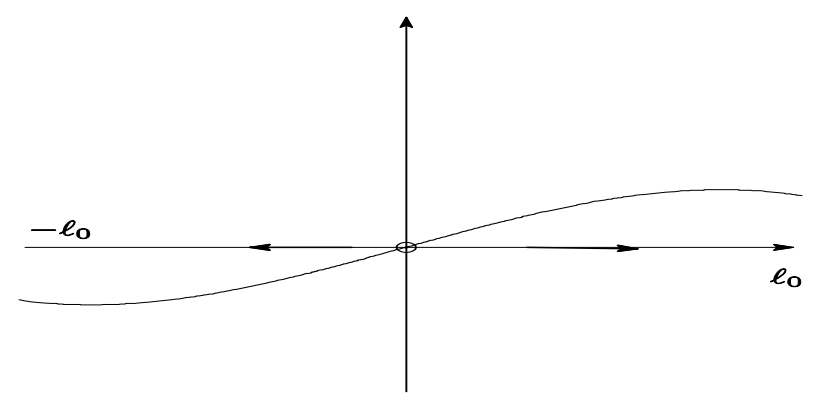

Figure 2. $\gamma=1$ and $1+\left(a_{3}^{0} \ell_{0}{ }^{2} h_{3} N_{2}\right)^{-1} \leq K_{1} v$. $g_{+}(\zeta)<\zeta^{2 \gamma}-1+\zeta^{\gamma-1} v K_{\gamma}$. Because $\zeta^{2 \gamma}-1<0$ and $\zeta^{\gamma-1} v K_{\gamma}<0$ on $(-1,0)$, we see that $g_{+}$has a unique zero point $\zeta_{0}$ on $(-1,1)$. Clearly, $g_{-}$has only one zero $-\zeta_{0}$, because $g_{+}(\zeta)=g_{-}(-\zeta)$ for all $\zeta,-1 \leq \zeta \leq 1$, which proves the assertion. The sign of $\tilde{f}(z) / z$ is almost clear from the definitions of $g_{ \pm}, g_{-}$ and the argument in the above.

Step 3. We will show the asymptotic formula of $z_{0}$ in (3.4). In view of the argument in Step 2 we may consider $g_{+}(\zeta)=0$.

If we set $\zeta=1+\xi,(-1<\xi \leq 0)$, then we have

$$
\begin{aligned}
A_{\gamma} & =(1+\xi)^{2 \gamma}-1+v K_{\gamma}(1+\xi)^{\gamma-1} \\
& =2 \gamma \xi+v K_{\gamma}(1+(\gamma-1) \xi)+\psi(\xi),
\end{aligned}
$$

where $\psi(\xi)=O\left(\xi^{2}\right)$ is a polynomial of $\xi$ with positive coefficients. Hence we have

$$
\begin{aligned}
& \xi+\left(2 \gamma+(\gamma-1) v K_{\gamma}\right)^{-1} \psi(\xi) \\
= & \left(A_{\gamma}-v K_{\gamma}\right)\left(2 \gamma+(\gamma-1) v K_{\gamma}\right)^{-1} \\
= & : \Lambda .
\end{aligned}
$$

Hence, for $\Lambda$ sufficiently small we can uniquely solve (3.10). By an implicit function theorem we see that $\xi=\xi(\Lambda)$ is a smooth function of $\Lambda$ such that $\xi(0)=0, \xi^{\prime}(0)=1$. It follows that

$$
\begin{aligned}
\zeta & =z_{0} / \ell_{0}=1+\xi(\Lambda)+O\left(\Lambda^{2}\right) \\
& =1-v K_{\gamma}\left(2 \gamma+(\gamma-1) v K_{\gamma}\right)^{-1}+O\left(v^{2} K_{\gamma}^{2}, N_{2}^{-1}\right) .
\end{aligned}
$$

Therefore we have (3.4).

Step 4. Next we will prove (3.5). We will solve $g_{ \pm}(\zeta)=0$, namely

$$
\zeta^{2 \gamma}-1+v K_{\gamma} \zeta^{\gamma-1}=A_{\gamma}
$$

for $0<\zeta \leq 1$. Hence we have

$$
v K_{\gamma}=\zeta^{1-\gamma}-\zeta^{1+\gamma}+A_{\gamma} \zeta^{1-\gamma} .
$$

If $\zeta$ does not converges to zero when $v K_{\gamma} \rightarrow \infty$, then there exist $c>0$ and a sequence

$\zeta_{n} \geq c>0(n=1,2, \cdots)$. Since the right-hand side of (3.12) is bounded for $\zeta=\zeta_{n}$, this leads to a contradiction. Hence $v K_{\gamma}$ is asymptotically equal to $\zeta^{1-\gamma}\left(1+A_{\gamma}\right)$.

By solving this relation we have

$$
\zeta \sim\left(1+A_{\gamma}\right)^{1 / \gamma-1}\left(v K_{\gamma}\right)^{-1 / \gamma-1}
$$

when $v K_{\gamma} \rightarrow \infty$. By (3.11) we have $\zeta^{1-\gamma}=\left(1+A_{\gamma}\right)^{-1}\left(v K_{\gamma}+\zeta^{1+\gamma}\right)$. It follows that

$$
\begin{aligned}
\zeta & =\left(1+A_{\gamma}\right)^{1 /(\gamma-1)}\left(v K_{\gamma}+\zeta^{1+\gamma}\right)^{1 /(1-\gamma)} \\
& =\left(1+A_{\gamma}\right)^{1 /(\gamma-1)}\left(v K_{\gamma}\right)^{1 /(1-\gamma)} \times\left(1-\frac{\zeta^{1+\gamma}}{v K_{\gamma}(\gamma-1)}+\cdots\right) .
\end{aligned}
$$


By simple computations we obtain (3.5).

Step5. If $\gamma=1$, then we have

$g_{ \pm}(\zeta)=\zeta^{2} \pm v K_{1}-1-A_{1}$. We can easily see that $g_{-}(\zeta) \neq 0$ on $[-1,1]$. The solution of $g_{+}(\zeta)=0$ is given by $\pm \sqrt{1-v K_{1}+A_{1}}$. The rest of the assertion is almost clear from this formula. This completes the proof.

Remark. We will briefly discuss the difference of dynamics of $z$ in (3.1) for $f$ and $\tilde{f}$. We note that two functions are identical for $|z| \leq \ell_{0}-\eta$. For a small number $\eta>0$, consider the case shown in Figure 2 for $\gamma=1$. Then $f$ looks like as in Figure 3 where new attractive equilibrium points appear near $\pm \ell_{0}$ because we have made a modification to $a_{3}$ so that $\pm f\left( \pm \ell_{0}\right)<\tilde{f}$. The new equilibrium point corresponds to that of $\tilde{f}$ with modulus larger than $\ell_{0}$. Because of the new equilibrium points we have an apriori estimate of the solution for $f$. Namely, the orbit started from a neighborhood of the origin does not go beyond $\pm \ell_{0}$. This fact is important since, if otherwise, the efficiency $a_{3}(\underset{\sim}{z})$ becomes negative. Note that the dynamics of $f$ and $\tilde{f}$ is the same outside some neighborhood of the boundary $z= \pm \ell_{0}$. We also note that a similar situation occurs in the case $\gamma \geq 2$ with $z_{0}<\ell_{0}-\eta$. (cf. Figures 1 and 4). On the other hand, if $z_{0} \geq \ell_{0}-\eta$, then the dynamics of $f$ and $\tilde{f}$ in $z_{0}-z$ may be different, while in other part both are the same.

We also note that the apriori estimate holds for $f$. Therefore apart from the neighborhood of $z= \pm \ell_{0}$ the dynamics of $f$ is well approximated by that of $\tilde{f}$, for which $\tilde{f}$ we can make concrete analysis of the dynamics, although we do not have the apriori estimate.

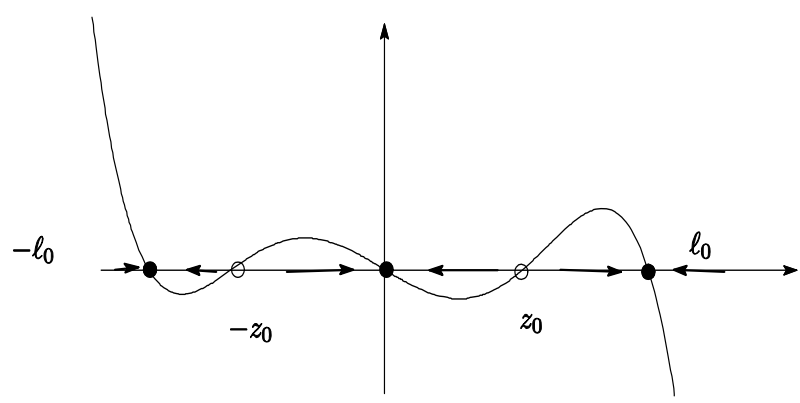

Figure 3. Picture of $f \cdot \gamma=1$.

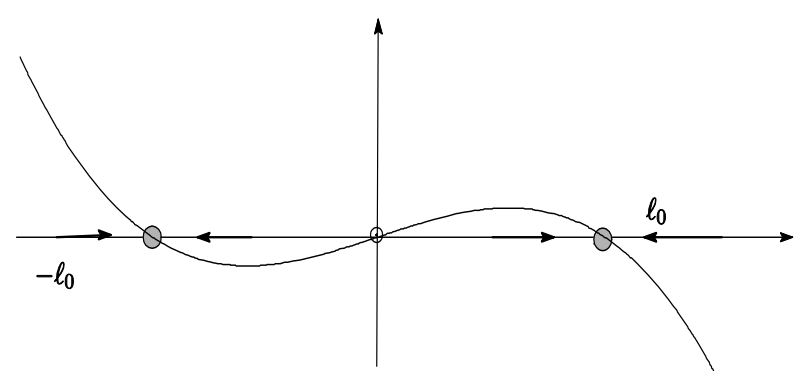

Figure 4. Picture of $f . \quad \gamma \geq 2$.
We will study behaviors of solutions under the effect of evolution.

\section{1) Behaviors near the equilibrium point.}

We consider how the dynamics of (1.1)-(1.4) is related to the dynamics (1.1)-(1.3) without evolution. We recall that (1.1)-(1.3) without an evolutional effect has what is called a tea-cup attractor. The isocline of (1.1)-(1.3), (3.1) is given by the family of equations

$$
\begin{gathered}
r\left(1-N_{1} / K\right)-\frac{a_{2} N_{2}}{1+a_{2} h_{2} N_{1}}=0, \\
\frac{c_{2} a_{2} N_{1}}{1+a_{2} h_{2} N_{1}}-d_{2}-\frac{a_{3} N_{3}}{1+a_{3} h_{3} N_{2}}=0, \\
\frac{c_{3} a_{3} N_{2}}{1+a_{3} h_{3} N_{2}}-d_{3}=0, \\
f(z)=0 .
\end{gathered}
$$

It follows from (3.15) that

$$
a_{3} N_{2}=\frac{d_{3}}{c_{3}-h_{3} d_{3}}=: A .
$$

One may assume that $A$ is small since $d_{3} \sim 0$ is small. By (3.14) we have

$$
a_{3} N_{3}=\left(1+h_{3} A\right)\left(\frac{c_{2} a_{2} N_{1}}{1+a_{2} h_{2} N_{1}}-d_{2}\right)=0 .
$$

Let us first consider the non-evolutional case, $z=0$ or the case where evolution becomes stationary, namely $z \rightarrow 0$. Then there exists $C>0$ such that $a_{3}(z)>C$. Hence $N_{2}=A / a_{3}$ is small and $N_{1}$ is close to $K$, by (3.13). It follows that there exists $C_{1}>0$ such that $\left(c_{2} a_{2} N_{1}\right) /\left(1+a_{2} h_{2} N_{1}\right)-d_{2}>C_{1}$ if $d_{2}$ is sufficiently small. In terms of (3.17) and (3.18) $N_{3} / N_{2}$ tends to infinity when $A \rightarrow 0$. This implies a typical behavior of $N_{2}$ and $N_{3}$ around an equilibrium point when there is little effect of evolution. Numerical experiments show that the decrease of $N_{3}$ occurs soon after the orbit approaches to the equilibrium point, namely $N_{3} / N_{2}$ becomes sufficiently large.

Let us consider the evolutional case. Then the main difference from the non-evolutional case is that $a_{3}(z)$ may tends to zero. For the sake of simplicity, let us consider the case $\gamma=1$ or $\gamma=2$. Assume that there exists an orbit such that $N_{3} \geq 1$ and $N_{3} / N_{2}$ grows large. Because $\sqrt{N_{3}} / N_{2}$ also grows large, it follows from (3.4) that $z_{0}$ tends to zero, and we are in the situation that the orbit of $z>z_{0}$ tends to $l_{0}$. Therefore $a_{3}(z)$ tends to zero. Hence the boundedness of $N_{2}$ implies that $\left(c_{3} a_{3} N_{2}\right) /\left(1+a_{3} h_{3} N_{2}\right)-d_{3}$ becomes negative. Therefore, by (1.3), $N_{3}$ exponentially decreases. Note that the decrease of $a_{3}(z)$ begins after $\sqrt{N_{3}} / N_{2}$ exceeds a certain constant independent of $\mathrm{N}_{2}$ and $N_{3}$ when 
$\gamma=1$. This exhibits a strong contrast to the non evolutional case where the collapse of $N_{3}$ occurs after $N_{3} / N_{2}$ becomes sufficiently large.

2) Effect of the parameter $\gamma$ to evolution.

In the predatory efficiency $a_{3}(z)$ in (C.1) $\gamma$ represents sensitivity to $z$. Namely, as $\gamma$ increases, $a_{3}(z)$ for small $z$ approaches to a constant function. The dynamics of $z$ is quite different in the cases $\gamma=1$ and $\gamma \geq 2$. Indeed, if $\gamma=1$ and

$$
1+\left(l_{0}^{2} a_{3}^{0} h_{3} N_{2}\right)^{-1} \leq K_{1} v=\frac{\sqrt{2 \delta}}{l_{0}^{2} h_{3} \sqrt{a_{0}^{3}}} \frac{\sqrt{N_{3}}}{N_{2}},
$$

then evolution progresses. (cf. Figure 2 and Figure 3). The latter condition means either the cost of evolution is small, $\delta \gg 1$ or $\sqrt{N_{3}} / N_{2} \gg 1$. We note that the attracttive equilibrium points near $z= \pm \ell_{0}$ have the effect to hold the orbits around $z= \pm \ell_{0}$. Conversely, if $1+\left(l_{0}^{2} a_{3}^{0} h_{3} N_{2}\right)^{-1} \geq K_{1} v$, then we see that fluctuations in progress and rest of evolution takes place.

In the case $\gamma \geq 2$ we have a different situation. Indeed, if $N_{3}<1 /\left(2 \gamma a_{3}^{0} \delta \ell_{0}^{2 \gamma-2}\right)$, then the evolution becomes stationary. If otherwise, then similar fluctuations in progress and rest of evolution as in the case $\gamma=1$ takes place. We will show in the next section that in the linear case $a_{3}(z)=\alpha z+\beta$ we have a sharp contrast to the case $\gamma \geq 2$.

3) Fluctuations of $N_{1}$ and $N_{2}$.

The rhythm of $N_{1}$ and $N_{2}$ is also observed in a non- evolutional system and it is related with the structure of a tea-cup attractor. We have a similar phenomenon for an evolutional system.

Let $\tilde{N}_{1}, \tilde{N}_{2}, \tilde{N}_{3}=0$ and $\tilde{z}$ be a solution of (1.1)(1.4). One can show by Poincaré -Bendixon theorem that $\tilde{N}_{1}$ and $\tilde{N}_{2}$ are an oscillating solution of two species under appropriate choice of parameters. Note that $\tilde{z}$ tends to zero exponentially. By the continuity of solutions of the initial value problem with respect to an initial value and the apriori estimate of a solution, one can see that for every $T>0$ and $\varepsilon>0$ there exists $\delta>0$ such that if

$$
\begin{aligned}
& \left|N_{1}(0)-\tilde{N}_{1}(0)\right|<\delta,\left|N_{2}(0)-\tilde{N}_{2}(0)\right|<\delta, \\
& N_{3}(0)<\delta,|z(0)-\tilde{z}(0)|<\delta,
\end{aligned}
$$

then

$$
\begin{aligned}
& \left|N_{1}(t)-\tilde{N}_{1}(t)\right|<\varepsilon,\left|N_{2}(t)-\tilde{N}_{2}(t)\right|<\varepsilon, \\
& N_{3}(t)<\varepsilon,|z(t)-\tilde{z}(t)|<\varepsilon,
\end{aligned}
$$

for all $0 \leq t \leq T$. Here, without loss of generality we may assume that the initial time is 0.Especially, this shows that there appears a rhythm of $N_{1}$ and $N_{2}$ for some interval of time. Note that $N_{3}$ is small and the evolution becomes stationary, i.e., $a_{3} \rightarrow a_{3}^{0}$.
In order to estimate $T$, we take $N_{1}(0)=\tilde{N}_{1}(0)$, $N_{2}(0)=\tilde{N}_{2}(0)$, and $z(0)=\tilde{z}(0)$. By integrating the equation of $N_{3}$, one has

$$
N_{3}(t)=N_{3}(0) \exp \left(-d_{3} t+\int_{0}^{t} \frac{c_{3} a_{3} N_{2}(s)}{1+h_{3} a_{3} N_{2}(s)} \mathrm{d} s\right) .
$$

Hence, if we have

$$
\left|-d_{3} t+\int_{0}^{t} \frac{c_{3} a_{3} N_{2}(s)}{1+h_{3} a_{3} N_{2}(s)} \mathrm{d} s\right| \leq \eta t, \quad 0 \leq t \leq T
$$

for sufficiently small $\eta$, then we have $N_{3}(t) \leq N_{3}(0) \mathrm{e}^{\eta T}<\varepsilon$, from which we have the estimate of time length $T, T<\eta^{-1} \ln \left(\varepsilon / N_{3}(0)\right)$. We have a similar condition like (3.19) in the general case $t_{0} \leq t \leq t_{0}+T$ by replacing 0 and $T$, respectively, by $t_{0}$ and $T_{0}+T$. A similar condition like (3.19) holds for some $t_{0}$ and $T$ if we have an averaging property:

$$
\lim _{t \rightarrow \infty} \frac{1}{t} \int_{0}^{t} \frac{c_{3} a_{3} N_{2}(s)}{1+h_{3} a_{3} N_{2}(s)} \mathrm{d} s=d_{3} .
$$

4) The limit case when evolution cost tends to zero.

We assume $N_{3}>0$. If the evolution cost tends to zero, namely $\delta$ grows from zero to $\infty$, then, by (3.4) and the definition of $K_{\gamma}, z_{0}$ approaches to the origin.

Therefore, the evolution progresses, namely $z$ approaches either of the points $\pm \ell_{0}$. It follows that the predatory efficiency $a_{3} \rightarrow 0$. By the same reasoning as in 1) $N_{3}$ tends to zero. We note that in the limit case $\delta \rightarrow \infty$ the third species dies out. This agrees with an ecological observation.

\section{Evolution for a Linear Predatory Efficiency}

We will discuss the evolution in the case

$$
a_{3}(z)=\left\{\begin{array}{l}
\alpha z+\beta, \text { if } \alpha z+\beta \geq 0 \\
0, \quad \text { if otherwise, }
\end{array}\right.
$$

where $\alpha \neq 0$ is a real constant and $\beta>0$.As in the previous case we make modifications of $a_{3}(z)$ in some small neighborhood of the zero point $\ell_{0}=-\beta / \alpha$ such that $a_{3} \in C^{1}(\mathbb{R})$.

For the sake of simplicity, we assume that $\alpha>0$. By repeating the same arguments as in Section 2 we see that the system of Equations (1.1)-(1.4) with the initial condition (1.6) has a unique global solution in $t_{0} \leq t<\infty$.

We will study the dynamics of the evolution in relation with the populations $N_{2}$ and $N_{3}$. We now define

$$
f(z):=-z / \delta-\alpha N_{3}\left(1+h_{3} N_{2}(\alpha z+\beta)\right)^{-2} .
$$

The condition $f(z) \geq 0$ is equivalent to 


$$
z\left(1+h_{3} N_{2}(\alpha z+\beta)\right)^{2}+\alpha \delta N_{3} \leq 0 .
$$

By definition we may consider (4.3) in the set $I:=\{z ; \alpha z+\beta \geq 0\}$ because, if otherwise, $f(z)=-z / \delta>0$. Set

$$
\phi(z):=z\left(1+h_{3} N_{2}(\alpha z+\beta)\right)^{2}
$$

and calculate the minimum of $\phi(z)$ in $I$. It is taken at $z=-\left(1+h_{3} N_{2} \beta\right) / 3 \alpha h_{3} N_{2}$ with the minimum value given by

$$
-4 \frac{\left(1+h_{3} N_{2} \beta\right)^{3}}{27 \alpha h_{3} N_{2}} \sim-4 \frac{\beta^{3} N_{2}^{2} h_{3}^{2}}{27 \alpha}+\mathrm{O}\left(N_{2}\right) .
$$

We recall that $f(z) \geq 0$ is equivalent to $\phi(z)+\alpha \delta N_{3} \leq 0$. Therefore, if

$-\alpha \delta N_{3}>-4 \beta^{3} N_{2}^{2} h_{3}^{2} /(27 \alpha)$ modulo terms of $\mathrm{O}\left(N_{2}\right)$, namely

$$
\sqrt{N_{3}} / N_{2}<c_{0} / \sqrt{\delta}, c_{0}=2 \beta \sqrt{\beta} h_{3} /(3 \alpha \sqrt{3}),
$$

then there appear an attractive equilibrium point $z_{0}$ near the origin $z=0$. This means that the predatory efficiency $a_{3}$ is close to a constant function if $\sqrt{N_{3}} / N_{2}$ is sufficiently small. Indeed, the equilibrium point $z_{0}$ can be estimated as

$$
z_{0}=-\frac{\alpha \delta}{\beta^{2} h_{3}^{2}}\left(\sqrt{N_{3}} / N_{2}\right)^{2}+\mathrm{O}\left(\left(\sqrt{N_{3}} / N_{2}\right)^{3}\right) .
$$

In view of the linearity of $a_{3}(z)$ and the smallness of $\sqrt{N_{3}} / N_{2}$ near the equilibrium point we see that $a_{3}(z)$ is almost constant for small changes of $\sqrt{N_{3}} / N_{2}$.

Suppose now that (4.4) does not hold. Then the attracttive equilibrium point near $z=0$ disappears, and there remains an attractive equilibrium point near the zero of $a_{3}$. Hence the evolution progresses and $a_{3}(z)$ tends to zero. This alternative between the rest and the progress of evolution shows a high contrast to the case of a convex predatory efficiency function discussed in the previous section.

\section{Behaviors of Solutions as Cost Increases}

In this section we study the convergence of a solution of an evolutional system to that of a non-evolutional one when the evolutional cost increases, namely $\delta$ decreases to zero. Let $N_{j}(t) \equiv N_{j}(t, \delta)$ and $z_{2}(t) \equiv z_{2}(t, \delta)$ be the solution of (1.1)-(1.4). Let $\tilde{N}_{j}(t)$ be the solution of the non-evolutional system (1.1)-(1.3), namely $a_{3}\left(z_{2}-\hat{z}_{2}\right) \equiv a_{3}^{0}$. Then we have

THEOREM 5.1. Assume (2.3). Let $T>0$ be arbitrarily given. Then we have

$$
\lim _{\delta \rightarrow 0} N(t, \delta)=\tilde{N}(t)
$$

uniformly in ton $t_{0} \leq t \leq T+t_{0}$.
Proof. By integrating (1.4) we have

$$
z_{2}-\hat{z}_{2}=-g \int_{t_{0}}^{t} \exp \left(-\frac{g}{\delta}(t-s)\right) \frac{N_{3}(s) a_{3}^{\prime}}{\left(1+a_{3} h_{3} N_{2}(s)\right)^{2}} \mathrm{~d} s .
$$

If we make the change of variables, $\sigma=g(t-s) / \delta$, then we have

$$
z_{2}-\hat{z}_{2}=-\delta \int_{0}^{t_{1}} \mathrm{e}^{-\sigma} w(\sigma) \mathrm{d} \sigma,
$$

where $t_{1}=g\left(t-t_{0}\right) / \delta$ and

$$
w(\sigma)=\frac{N_{3}\left(t-\delta g^{-1} \sigma\right) a_{3}^{\prime}}{\left(1+a_{3} h_{3} N_{2}\left(t-\delta g^{-1} \sigma\right)\right)^{2}} .
$$

Because $N_{3}\left(t-\delta g^{-1} \sigma\right)$ is uniformly bounded in $\delta$ by the apriori estimate, it follows that $e^{\sigma}$ times the integrand is uniformly bounded in $t \geq t_{0}$ when $\delta \rightarrow 0$. Hence, the modulus of the integral can be bounded by a constant times $\int_{0}^{\infty} e^{-\sigma} \mathrm{d} \sigma$. It follows that $z^{2}-\hat{z}_{2}^{2} \rightarrow 0$ uniformly in $t \geq t_{0}$ when $\delta \rightarrow 0$. This entails that $a_{3}\left(z^{2}-\hat{z}_{2}^{0}\right) \rightarrow a_{3}^{0}(\delta \rightarrow 0)$ uniformly in $t \geq t_{0}$.

For the sake of simplicity we write (1.1)-(1.3) in

$$
N(t) \equiv N(t, \delta)=N^{0}+\int_{t_{0}}^{t} F(N(s, \delta)) \mathrm{d} s,
$$

where we use the same notation $F$ as in (2.18). Since

$$
N(t, \delta)-\tilde{N}(t)=\int_{t_{0}}^{t} F(N(s, \delta))-F(\tilde{N}(s)) \mathrm{d} s,
$$

we have

$$
|N(t, \delta)-\tilde{N}(t)| \leq \int_{t_{0}}^{t}|F(N(s, \delta))-F(\tilde{N}(\mathrm{~s}))| \mathrm{d} s,
$$

where the absolute value of a vector means the norm of a vector. Because we have the uniform estimate of $N(s, \delta)$ in $\delta$ by (1) of Remark in Section 2, we have

$$
\begin{aligned}
& |F(N(s, \delta))-F(\tilde{N}(s))| \\
& \leq K_{1}\left(|N(s, \delta)-\tilde{N}(s)|+\left|a_{3}-a_{3}^{0}\right|\right),
\end{aligned}
$$

for some $K_{1}>0$ independent of $\delta$.

By (1.5) and (5.2) we have

$$
a_{3}\left(z_{2}-\hat{z}_{2}\right)-a_{3}^{0}=-a\left(-\delta \int_{0}^{t_{1}} \mathrm{e}^{-\sigma} w(\sigma) \mathrm{d} \sigma\right) .
$$

Because $a(0)=0$, there exists a constant $K_{2}>0$ independent of $\delta$ such that the right hand side of (5.4) can be estimated by $K_{2} \delta$. It follows that for any $\varepsilon>0$ there exist $\delta_{0}>0$ and $A>0$ such that, for $0<\delta<\delta_{0}$

$$
|F(N(s, \delta))-F(\tilde{N}(s))| \leq A|N(s, \delta)-\tilde{N}(s)|+\varepsilon .
$$

Therefore we have

$$
|N(t, \delta)-\tilde{N}(t)| \leq \varepsilon T+A\left|\int_{t_{0}}^{t}\right| N(\sigma, \delta)-\tilde{N}(\sigma)|\mathrm{d} \sigma| .
$$


By Gronwall's inequality we obtain, for $0<\delta<\delta_{0}$

$$
|N(t, \delta)-\tilde{N}(t)| \leq \varepsilon T \exp \left(A\left|t-t_{0}\right|\right)
$$

Because $\varepsilon>0$ is arbitrary, we have the desired estimate.

\section{Discussion}

Evolutional Lotka-Volterra system does not seem to be well understood analytically except for the case of two species. In this paper, we have studied how the evolutional change of a character influences global behaviors of a Lotka-Volterra system for three species. We introduced an evolutional equation based on a quantitative genetic model into a Lotka-Volterra system of equations and we proved the existence and the uniqueness of a global solution as well as apriori estimates of a solution. By virtue of these properties, we have given analytical proofs of properties which are different from the nonevolutional system. We hope that some of the properties shown in this paper hold for more general food web settings. It is also interesting to make numerical analysis of our theory in order to understand the effect of evolution. The study of these problems will be left for the future study.

\section{REFERENCES}

[1] R. Lande, "Quantitative Genetic Analysis of Multivariate Evolution Applied to Brain: Body Allometry," Evolution, Vol. 33, No. 1, 1979, pp. 402-416. http://dx.doi.org/10.2307/2407630

[2] R. Lande and S. J. Arnold, "The Measurement of Selection on Correlated Characters," Evolution, Vol. 37, No. 6, 1983, pp.1210-1226. http://dx.doi.org/10.2307/2408842

[3] P. A. Abrams and H. Matsuda, "Prey Adaptation as a Cause of Predator-Prey Cycles," Evolution, Vol. 51, No. 6, 1997, pp. 1742-1750. http://dx.doi.org/10.2307/2410997

[4] P. A. Abrams, "Evolutionay Responses Offoraging-Related traits in Unstable Predator-Prey Systems," Evolutionary Ecology, Vol. 11, No. 6, 1997, pp. 673-686. http://dx.doi.org/10.1023/A:1018482218068

[5] P. A. Abrams and H. Matsuda, "Fitness Minimization and Dynamic Instability as a Consequence of Predator-Prey Coevolution," Evolutionary Ecology, Vol. 11, No. 1, 1997, pp. 1-20. http://dx.doi.org/10.1023/A:1018445517101

[6] Y. Takeuchi, "Global Dynamical Properties of LotkaVolterra Systems," World Scientific, Singapore, 1996.

[7] R. A. Fisher, "The Genetical Theory of Natural Selection," Claredon Press, Oxford, 1930. 


\section{Appendix}

(A) The following lemma is used in Section 5 .

LEMMA. (Gronwall) Let $I=[a, b] \subseteq \mathbb{R}$ be $a$ closed interval and let $s \in I$. Let $u$ be continuously differentiable in I such that, for some constants $c \geq 0$ and $A \geq 0$ the inequality

$$
0 \leq u(t) \leq c+A\left|\int_{s}^{t} u(\sigma) \mathrm{d} \sigma\right|
$$

holds true for $t \in I$. Then we have $u(t) \leq c \exp (A|t-s|)$ on $I$.

Proof. For the sake of simplicity, we consider the case $t \geq s$. Denoting the right hand side of the inequality by $v(t)$ we have the relations, $u(t) \leq v(t), v(s)=c$ and

$\dot{v}(t)=A u(t) \leq A v(t)$. Multiplying the last inequality with $\mathrm{e}^{-A(t-s)}$, we have $(\mathrm{d} / \mathrm{d} t)\left(\mathrm{e}^{-A(t-s)} v(t)\right) \leq 0$. By integration we get $u(t) \leq v(t) \leq c \mathrm{e}^{A t-s \mid}$.

(B) We will briefly show how to deduce (1.4) from the theory of quantitative genetics. Let $z=z_{2}-\bar{z}_{2}, g$ and $w$ be the average character value, the additive genetic variance and the average adaptability of the character value $z$, respectively. Following the quantitative genetical model we have (cf. [1] and [2]).

$$
\mathrm{d} z / \mathrm{d} t=g(\partial / \partial z) \log W(z) .
$$

The left-hand side is the speed of evolution of a character value. Following Fisher, [7] we have $W=\mathrm{e}^{r}$. We assume that (cf. [3-5])

$$
\begin{gathered}
r(z)=N_{2}^{-1}\left(\mathrm{~d} N_{2} / \mathrm{d} t\right)+\log W_{2}, \\
W_{2}=\exp \left(-z^{2} / 2 \delta\right),
\end{gathered}
$$

where $\delta$ is the cost of evolution. By definition we have

$$
\frac{\partial}{\partial z}\left(\frac{1}{N_{2}} \frac{\mathrm{d} N_{2}}{\mathrm{~d} t}\right)=\frac{\partial}{\partial z}\left(\frac{c_{2} a_{2} N_{1}}{1+h_{2} a_{2} N_{1}}-d_{2}-\frac{a_{3} N_{3}}{1+a_{3} h_{3} N_{2}}\right) .
$$

Here $a_{2}, h_{2}$ and $d_{2}$ are certain constants. We have

$$
\frac{a_{3} N_{3}}{1+a_{3} h_{3} N_{2}}=\frac{N_{3}}{N_{2}}-\frac{N_{3}}{N_{2}} \frac{1}{1+a_{3} h_{3} N_{2}} .
$$

Because one may regards $N_{j}$ as a constant function when $z$ varies, the right-hand side of (7.1) can be replaced by

$$
\frac{N_{3}}{N_{2}} \frac{a_{3}^{\prime} N_{2}}{\left(1+a_{3} h_{3} N_{2}\right)^{2}}=-\frac{N_{3} a_{3}^{\prime}}{\left(1+a_{3} h_{3} N_{2}\right)^{2}} .
$$

Hence we obtain (1.4). 